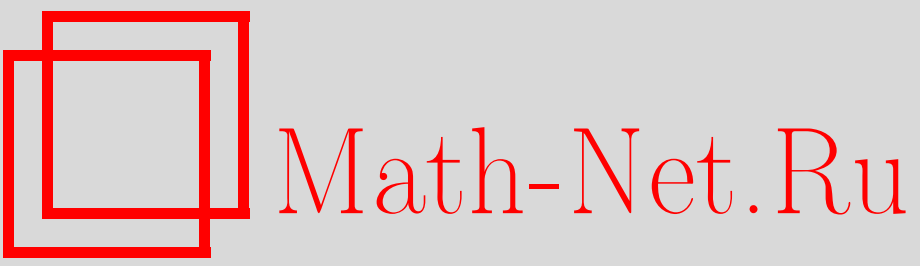

С. А. Богатый, Компактная однородность универсального метрического пространства Урысона, УМН, 2000, том 55, выпуск 2, 131-132

DOI: https://doi.org/10.4213/rm273

Использование Общероссийского математического портала Math-Net.Ru подразумевает, что вы прочитали и согласны с пользовательским соглашением

http://www . mathnet.ru/rus/agreement

Параметры загрузки:

IP : 54.198 .64 .247

26 апреля 2023 г., 12:31:19 


\title{
КОМПАКТНАЯ ОДНОРОДНОСТЬ УНИВЕРСАЛЬНОГО МЕТРИЧЕСКОГО ПРОСТРАНСТВА УРЫСОНА
}

\author{
С. А. БОГАТЫЙ
}

В 1998 году отмечался 100-летний юбилей П. С. Урысона. Это возродило интерес к его оригинальным работам. В одной из своих последних работ П. С. Урысон построил и изучил универсальное метрическое пространство $(U, \rho)$. Как отмечает А. М. Вершик: "Эта работа не получила широкой известности, хотя того заслуживает". П.С. Урысон показал, что существует полное сепарабельное метрическое пространство $U$, обладающее свойствами:

(1) $\left(U_{\mathscr{S}}\right)$ всякое сепарабельное метрическое пространство может бъть изометрично отображено в $U$;

(2) $\left(\right.$ НF $\left._{\mathscr{F}}\right)$ всякая изометрия конечного подмножсества пространства $U$ может быть продолжена до изометрии всего пространства $U$.

П. С. Урысон показал также, что свойства (1) и (2) определяют пространство U с точностью до изометрии однозначно. Хотя свойства (1) и (2) независимы, они не образуют "оптимальное" описание пространства $U$ - ни с точки зрения минимальности условий для единственности, ни с точки зрения максимальности свойств пространства.

Будем говорить, что метрическое пространство $Y$ является (слабым) абсолютным метрическим әкстензором для класса метрических пространств $\mathscr{K}(Y \in(\mathrm{W}) \operatorname{AME}(\mathscr{K}))$, если для всякого пространства $X \in \mathscr{K}$ и всякой изометрии $f: A \rightarrow Y$, заданной на (замкнутом) подмножестве $A \subseteq X$, существует такая изометрия $F: X \rightarrow Y$, что $\left.F\right|_{A}=f$. Несложно убедиться, что фактически Урысон доказал следующую теорему единственности.

Теорема 1. Если $Y$ - полное сепарабельное метрическое пространство и $Y \in \operatorname{AME}(\mathscr{F})$, то $Y$ изометрично пространству Урысона $U$.

В доказательстве теоремы единственности П. С. Урысон пользуется свойством $\left(H_{\mathscr{F}}\right)$, однако как в оригиналной работе, так и у А.М. Вершика, в формулировке требуется более слабое свойство однородности. Заполнению указанного пробела служит

Теорема 2. Если метрическое пространство $Y$ обладает свойством $\left(U_{\mathscr{F}}\right)$ и для любых конечных изометричных подмножеств $A, B \subset Y$ существует такая изометрия (не обязательно на) $H: Y \rightarrow Y$, что $H(A)=B$, то $Y \in \operatorname{AME}(\mathscr{F})$.

П.С. Урысон ставит вопросы: "Не обладает ли пространство $U$ более сильным свойством однородности? Отрицательный ответ на этот вопрос до некоторой степени дается следующим ... Остается открытым вопрос: нельзя ли любые два конгруэнтных счетных множества в $U$ перевести друг в друга изометрическим отображением пространства $U$ на себя?"

ПрЕДЛОжЕниЕ 1. В пространстве $U$ существуют такие два счетные конгруэнтнье подмножества $A$ и $B$, что для всякого гомеоморфизма на $F: U \mapsto U F(A) \backslash B \neq \varnothing$.

Положительный ответ на первый вопрос Урысона дает

Теорема 3. Для всякого компактного подмножества $A \subset U$ и всякой изометрии $f: A \hookrightarrow U$ существует такая изометрия на $F: U \mapsto U$, что $\left.F\right|_{A}=f$.

Доказательство получается повторением рассуждений Урысона с заменой в его доказательстве ссылки на теорему 1 ссылкой на следующую лемму.

ЛЕмма. Для всякого вполне ограниченного метрического пространства $X$, всякой его точки $a \in X$ и всякой изометрии $f: X \backslash a \hookrightarrow U$ существует такая изометрия $F: X \hookrightarrow U$, чmо $\left.F\right|_{X \backslash a}=f$.

Работа выполнена при поддержке Российского фонда фундаментальных исследований (грант № 97-01-00357) и INTAS (грант № 96-0712). 
ДокАЗАтЕльство. Пусть $\varepsilon=\rho(a, X \backslash a)>0$ и $K_{\delta} \subset X \backslash a$-некоторая конечная $\delta$-сеть для $\delta \leqslant \varepsilon / 2$. Вложим метрическое пространство $K_{\delta} \cup a$ (с индуцированной метрикой) в $U$; можно считать, что это вложение $f_{\delta}: K_{\delta} \cup a \rightarrow U$ является продолжением вложения $\left.f\right|_{K_{\delta}}$.

Предположим, что для всех $k \leqslant n$ уже построены такие изометрии $f_{\delta / 2^{k}}: K_{\delta / 2^{k}} \cup$ $a \rightarrow U$, что $\left.f_{\delta / 2^{k}}\right|_{K_{\delta / 2^{k}}}=\left.f\right|_{K_{\delta / 2^{k}}}$ и $\rho\left(f_{\delta / 2^{k}}(a), f_{\delta / 2^{k-1}}(a)\right)=\delta / 2^{k-2}$. Пусть теперь $K_{\delta / 2^{n+1}}$ - такая конечная $\delta / 2^{n+1}$-сеть, что $K_{\delta / 2^{n}} \subseteq K_{\delta / 2^{n+1}}$. На множестве $K_{\delta / 2^{n+1}} \cup$ $a \cup f_{\delta / 2^{n}}(a)$ определим метрику. На $K_{\delta / 2^{n+1}} \cup a$ метрика индуцируется из $X$; на $K_{\delta / 2^{n+1}} \cup$ $f_{\delta / 2^{n}}(a)$ - из $U ; \rho\left(a, f_{\delta / 2^{n}}(a)\right)=\delta / 2^{n-1}$. Вложим метрическое пространство $K_{\delta / 2^{n+1}} \cup$ $a \cup f_{\delta / 2^{n}}(a)$ в $U$; для вложения $f_{\delta / 2^{n+1}}$ можно считать, что $\left.f_{\delta / 2^{n+1}}\right|_{K_{\delta / 2^{n+1}}}=\left.f\right|_{K_{\delta / 2^{n+1}}}$ и $f_{\delta / 2^{n+1}}\left(f_{\delta / 2^{n}}(a)\right)=f_{\delta / 2^{n}}(a)$. Предельная точка последовательности Коши $\left\{f_{\delta / 2^{n}}(a)\right\}$ и является искомой точкой $F(a)$.

СледствиЕ 1. Для сепарабельного метрического пространства Y следующие условия әквивалентны:

(1) $Y$ изометрично $U$;

(2) $Y$ полно и $Y \in \operatorname{AME}(\mathscr{F})$;

(3) $Y \in \operatorname{AME}(\mathscr{C})$, где $\mathscr{C}$ означает класс всех метрических компактных пространств.

СЛЕДСтвИЕ 2. Для всякого компактного подмножества $A \subset U$ dиаметра d cyществует такое топологическое вложение гильбертова куба $F: Q \rightarrow U$, что $A \subseteq F(Q)$ и $\operatorname{diam} F(Q)=d$.

ДоказАТЕльство. Вложим компакт $A$ в гильбертов куб $Q$. Согласно теореме Хаусдорфа метрику (индуцированную из $U$ ) с $A$ можно продолжить до метрики на $Q$. При этом можно считать, что $\operatorname{diam} Q=\operatorname{diam} A$. Согласно теореме Урысона $\left(U_{\mathscr{S}}\right)$ гильбертов куб в новой метрике изометрично вкладывется в $U$. Согласно теореме 3 можно при этом предполагать, что $A$ как подмножество $Q$ отображается на $A$ как подмножество $U$ тождественно.

СлеДСтвиЕ 3. Для всякого бикомпактного подмножества $A \subset X$ вполне регулярного пространства $X$ и всякого непрерьвного отображения $f: A \rightarrow U$ существует непрерывное продолжение $F: X \rightarrow U$.

Урысон доказал, что пространство U линейно связно и локально линейно связно. Согласно теореме Куратовского-Дугунджи из следствия 3 вытекает, что пространство Урысона $U$ связно и локально связно во всех размерностях. При этом из возможности продолжать без увеличения диаметра вытекает даже, что всякий шар (как открытый, так и замкнутый) в $U$ всякого положительного радиуса связен во всех размерностях.

Из теоремы 3 легко вывести, что пространство Урысона $U$ удовлетворяет свойству дизъюнктности дисков (DDP): Для любого компакта $X \subset U$ и любого $\varepsilon>0$ существует такой гомеоморфизм на $H: U \mapsto U$, что $\rho(a, H(a))=\varepsilon$ для всякой точки $a \in U$ u $X \cap H(X)=\varnothing$. Это показывает, что, вероятно, пространство $U$ гомеоморфно гильбертову простран$c m в$ y $\ell_{2}$. Из следствия 3 легко получить, что пространство $U$ является абсолютным экстензором для класса локально компактных паракомпактных пространств, однако вопрос о возможсности продолжения изометрии на $U$ с (замкнутого) локально компактного подмножества открыт. Из теоремы 3 вытекает также, что группа изометрий Iso $(U)$ пространства Урысона U содержит свободную группу от двух (а значит, и от счетного множества) образующих.

Автору приятно выразить свою благодарность Н. Бродскому и Н. Фоменко за ценные беседы.

Московский государственный

Принято редколлегией университет им. М. В. Ломоносова 26.01.2000

E-mail: bogatyi@mech.math.msu.su 